\title{
https://helda.helsinki.fi
}

\section{Wide field of view 3D label-free super-resolution imaging}

\section{Nolvi, Anton}

SPIE - the international society for optics and photonics 2018-02

Nolvi , A , Laidmae , I , Maconi , G , Heinämäki , J , Haeggström , E \& Kassamakov , I 2018 , Wide field of view 3D label-free super-resolution imaging . in Y G Soskind \& C Olson (eds), Photonic Instrumentation Engineering V ., UNSP 1053912 , Proceedings of SPIE, vol. 10539 , SPIE - the international society for optics and photonics, Washington, Conference on Photonic Instrumentation Engineering V , San Francisco , Canada , 30/01/2018 . https://doi.org/10.1117/12.2289

http://hdl.handle.net/10138/312000

https://doi.org/10.1117/12.2289120

unspecified

acceptedVersion

Downloaded from Helda, University of Helsinki institutional repository.

This is an electronic reprint of the original article.

This reprint may differ from the original in pagination and typographic detail.

Please cite the original version. 


\title{
Wide field of view 3D label-free super-resolution imaging
}

\author{
Anton Nolvi ${ }^{\mathrm{a}, \mathrm{b}}$, Ivo Laidmäe, d, Göran Maconi ${ }^{\mathrm{a}}$, Jyrki Heinämäki ${ }^{\mathrm{c}}$, \\ Edward Hæggström ${ }^{\mathrm{a}}$ and Ivan Kassamakov*a,e
}

\begin{abstract}
${ }^{a}$ University of Helsinki, Physics Department, Electronics Research Laboratory, Gustaf Hällströmin katu 2a, 00560 Helsinki, Finland; '̊̊̊̉bo Akademi, Faculty of Science and Engineering, Pharmaceutical Sciences Laboratory, Tykistökatu 6A, 20520 Turku, Finland; 'University of Tartu, Institute of Pharmacy, Faculty of Medicine, Nooruse 1, 50411 Tartu, Estonia; 'University of Tartu, Institute of Biomedicine and Translational Medicine, Department of Immunology, Faculty of Medicine, Ravila 19, 50411 Tartu, Estonia; ${ }^{\circ}$ Helsinki Institute of Physics, Gustaf Hällströmin katu 2a, 00560 Helsinki, Finland
\end{abstract}

\begin{abstract}
Recently, 3D label-free super-resolution profilers based on microsphere-assisted scanning white light interferometry were introduced having vertical resolution of few angstroms $(\AA)$ and a lateral resolution approaching $100 \mathrm{~nm}$. However, the use of a single microsphere to generate the photonic nanojet (PNJ) limits their field of view. We overcome this limitation by using polymer microfibers to generate the PNJ. This increases the field of view by order of magnitude in comparison to the previously developed solutions while still resolving sub $100 \mathrm{~nm}$ features laterally and keeping the vertical resolution in $1 \mathrm{~nm}$ range. To validate the capabilities of our system we used a recordable Blu-ray disc as a sample. It features a grooved surface topology with heights in the range of $20 \mathrm{~nm}$ and with distinguishable sub $100 \mathrm{~nm}$ lateral features that are unresolvable by diffraction limited optics. We achieved agreement between all three measurement devices across lateral and vertical dimensions. The field of view of our instrument was $110 \mu \mathrm{m}$ by $2 \mu \mathrm{m}$ and the imaging time was a couple of seconds.
\end{abstract}

Keywords: large field of view, super-resolution, label-free

\section{INTRODUCTION}

The development of imaging instruments capable of seeing nanometer scale structures has shifted research more and more into studying the nature in its very fundamental molecule and atomic level. The ability to see even the finest structures of cells has boosted the understanding of how to treat diseases. Furthermore a research of building artificial structures using nanometer sized building blocks, has led to ever growing field of nanotechnology creating wealth through new technological innovations.

Optically the imaging of small features is classically limited by the diffraction limit. ${ }^{1}$ This limit, generally described spatially as wavelength $\lambda$ divided by numerical aperture $N A$ of the imaging system, in practice limits the resolution to few hundreds of nanometers, depending of the resolution definition. ${ }^{2}$ The resolution can be increased by lowering the wavelength, as with scanning electron microscope (SEM), but the limit can also be overcome with light using various super-resolution techniques such as stimulated emission depletion (STED) and scanning near-field optical microscope $(\mathrm{SNOM}) ., 4$

The current super-resolution imaging techniques however all have limitations. They either require sample preparations through labeling or their field of view (FOV) is limited to point measurements. Furthermore the techniques require sophisticated instruments that are both expensive to buy and require special expertise to use.

A new approach to overcome optical diffraction barrier, where micro sized objects are used as additional magnification elements in close approximate of the sample. The object can focus light into beyond diffraction limit tight shape, which can be used to image nanometer sized features. This phenomenal, called photonic nanojet (PNJ), has been experimentally

*ivan.kassamakov@ helsinki.fi; phone +358 504486249 
proved by various groups to work as method to produce super-resolution $2 \mathrm{D}$ images. ${ }^{5,6} \mathrm{We}$ have shown that the PNJ can also be harnessed to bring optical interferometry 3D imaging technique into super-resolution domain in all of the dimensions using microspheres. ${ }^{7,8}$

The strength of the PNJ super-resolution imaging method is its ease of use, as it requires no sample preparations and offers large field of view. Many groups have used microsphere as PNJ creating objects, but it has been shown that the object shape is not limited to spheres ${ }^{9}$ and the field of view of single image can be enlarged even further.

\section{METHODS}

We combine scanning white light interferometry (SWLI) with PNJ creating fiber object into a label-free imaging method that can achieve vertical resolving power of sub-nanometers ${ }^{10}$ together with $100 \mathrm{~nm}$ lateral resolution. The method surpasses the diffraction barrier of imaging with conventional optics while still maintaining imaging features such as large field of view. Optical simulations of the sample interaction with the PNJ creating fiber were conducted to support the experimental observations.

\subsection{Measurement setup}

Our measurement instrument is based on SWLI. SWLI gives the high lateral resolving power of sub-nanometers while the horizontal resolution is enhanced with PNJ.

We use basic side-illuminated microscope frame (Nikon L-UEPI EPI) working with halogen light source (7724, $12 \mathrm{~V} / 100 \mathrm{~W}$, Philips) to provide broad spectrum illumination to the sample. The image is captured with CMOS camera (Orka Flash 2.8, Hamamatsu) through 1x magnification tube lens ( $\mathrm{f}=200 \mathrm{~mm}$, Nikon). The image is formed by using $50 \mathrm{x}$ magnification Mirau-type interferometer objective (CF IC EPI Plan DI 50x, NA 0.55, FOV 180x130 $\mu \mathrm{m}$ with 2/3" sensor, Nikon). The effective FOV of the setup without PNJ is 139x105 $\mu \mathrm{m}$. The optical components of the system are optimized for visible light.

The light through the objective is focused to the sample through the PNJ creating fiber, which was placed on the sample surface. The objective is moved along the optical axis to scan through sample topology variations with piezo controller (Pifoc P-721.CDQ, Pi). The optical schematic of the setup is illustrated in figure 1.

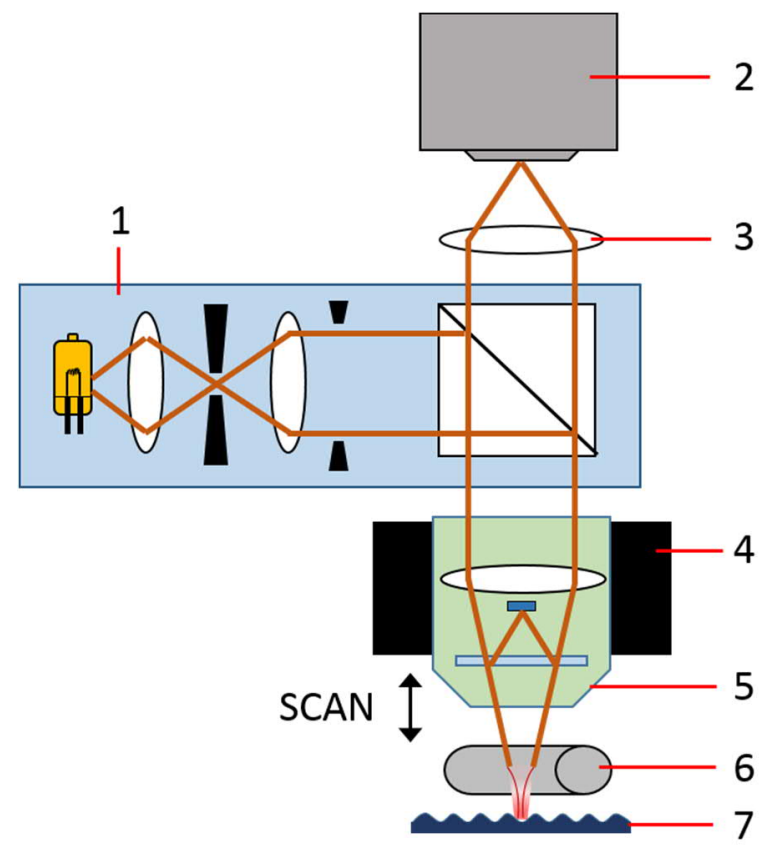

Figure 1. Graphical illustration of the optical arrangement of the measurement system: 1 - microscope frame, 2 - camera, 3 tube lens, 4 - piezo scanner, 5 - Mirau interferometer objective, 6 - PNJ fiber, and 7 - sample. 
During the scan the fiber to sample distance is remained stationary and only the fiber to objective distance is varied. A stack of images from equally spaced height distances records the interference data (intensity as function of sample-toobjective distance) that can be used to calculate 3D image of the sample topology. ${ }^{11}$

As the overall height span of the sample topology is limited to only few tens of nanometers, we use algorithm that requires only four recorded images from the interference pattern. ${ }^{12}$ The overall scanning time needed is therefore only few seconds, formed by the exposure, and the piezo movement and settle time. The processing time for calculations to generate 3D data is in the same rate as the actual scan.

A commercially available 3D data analysis software (Mountains Map 7.2, Digital Surf) was used to analyze the generated 3D data.

\subsection{Fibers}

Specially manufactured electrospun fibers were used as PNJ creating object. All fibers were produced with polycaprolactone (PCL, average Mw 80,000, cat. 440744, Lot\# MKBX1062V, Sigma-Aldrich) by melt-electrospinning, figure 2. PCL pellets were placed directly into a metal syringe $(10 \mathrm{ml})$ capped with a blunt metal needle $(21 \mathrm{G})$ which was placed in a heating nozzle system (NanoNC, South Korea). The system was connected to an oil circulator thermostat.
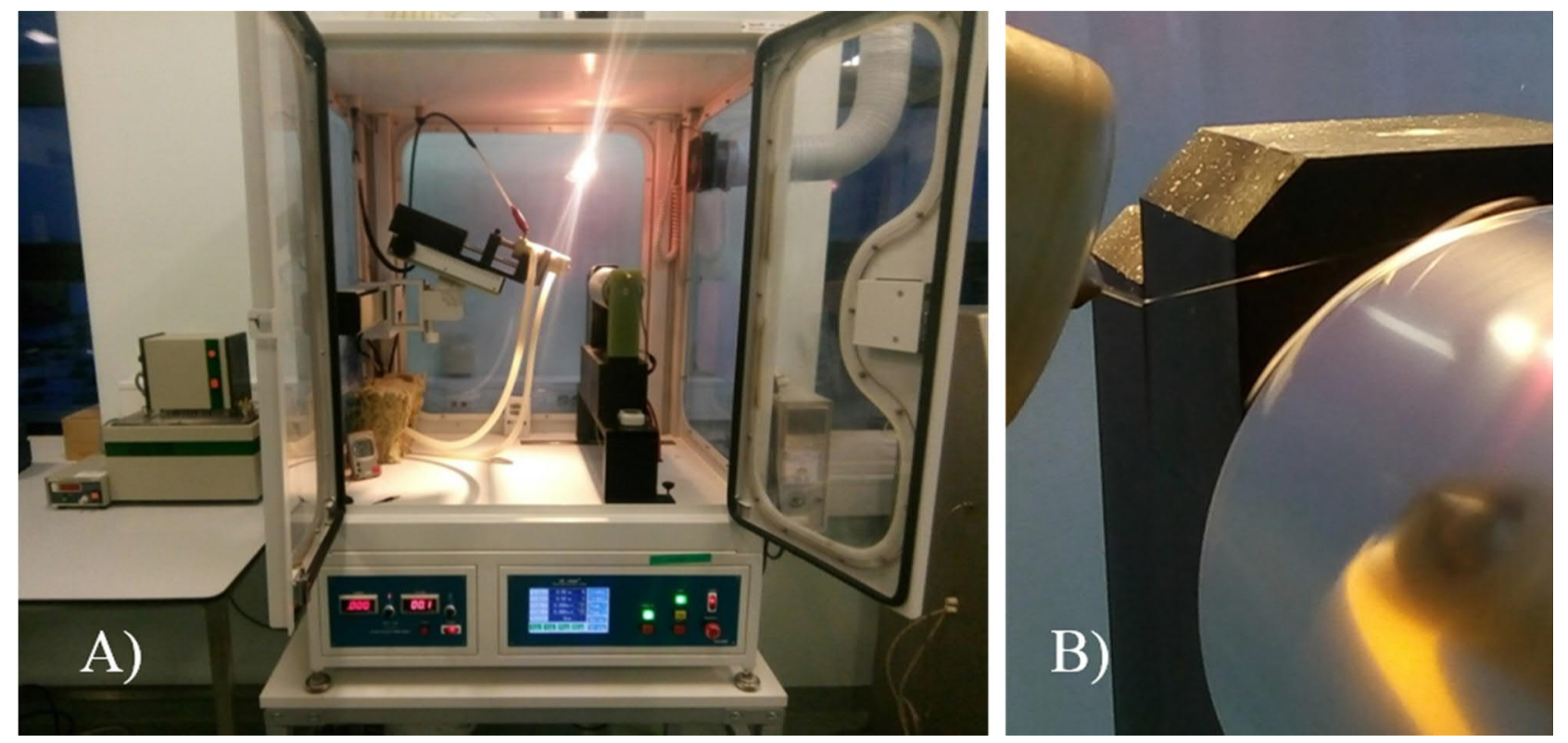

Figure 2. Melt-electrospinning set-up for preparing polymeric fibers. (A) Melt-electrospinning cabinet with an oil thermostat, and (B) Collection of the fibers on a rotating drum.

PCL was heated to $250{ }^{\circ} \mathrm{C}$ with a heating nozzle and the syringe was held upright to remove air bubbles from a polymer melt. A melt-electrospinning apparatus (NanoNC, South Korea) consisted of a programmable syringe pump connected to a syringe plunger which fed the molten polymer at a desired flow rate through a blunt needle. The system had also a highvoltage, direct current(HVDC) power supply, which was connected to the needle for creating an electric field across an air gap to a grounded collector. The feeding system was equipped with an $\mathrm{x}$-axis robotized slider to control the movement of the spinning system and fiber deposition on the collector.

The polymeric fibers were collected on a grounded rotating drum collector $(\varnothing 90 \mathrm{~mm})$ covered with aluminum foil. The critical process parameters in melt-electrospinning were set as follows: voltage $(5 \mathrm{kV})$, a collection distance $(50 \mathrm{~mm})$, flow rate $(1 \mathrm{ml} / \mathrm{h})$, a melting temperature $\left(250^{\circ} \mathrm{C}\right)$, and a collector rotation speed $(30 \mathrm{rpm})$. 


\subsection{Simulations}

To determine the near field imaging properties of thin glass fibers, we simulated a dielectric cylinder with a diameter of $11 \mu \mathrm{m}$, and refractive index of 1.47. The simulation was performed in the FDTD (Finite-Difference Time-Domain method) simulation software Rsoft FullWAVE, with a domain of $16 \mu \mathrm{m}(\mathrm{x}) * 10 \mu \mathrm{m}(\mathrm{y}) * 15.5 \mu \mathrm{m}(\mathrm{z})$ and a step size of $0.02 \mu \mathrm{m}$ in all directions. All boundaries use the perfectly matched layer (PML) model. The simulation was excited by a continuous plane wave $(\lambda=660 \mathrm{~nm})$ traversing in the negative $\mathrm{z}$ direction, and run until a steady state was achieved. The cylinder was placed at $6.5 \mu \mathrm{m}$ from the top of the simulation, with its axis pointing in the y direction.

\subsection{Samples}

A prepared recordable blue-ray disc (BD-R) was used as tightly specified sample. It features tightly spaced grooves that the laser of the optical drive tracks. The periodical groove dimensions are nominally specified as having pitch of $320 \mathrm{~nm}$ .${ }^{13}$ The groove depth is tens of nanometers. ${ }^{7,8,14}$ The bottom part of the groove is $100 \mathrm{~nm}$ and top part $200 \mathrm{~nm}$ wide. ${ }^{14}$ These dimensions are in a nanometer range that cannot be resolved with conventional diffraction limited optics.

The BD-R disc was prepared before measurement by first writing data on it. The optical drive writes data along the grooves creating changes in the reflectivity. The border of written data can be clearly seen even with conventional optics. It hence can be used an orientation indicator of the groove structure.

The BD-R disc features thick protective layers on top of the actual data layer where the laser tracking grooves are, figure 3. This thick protecting top part was manually peeled off prior measurement to expose the grooved surface structure for undistorted optical access.

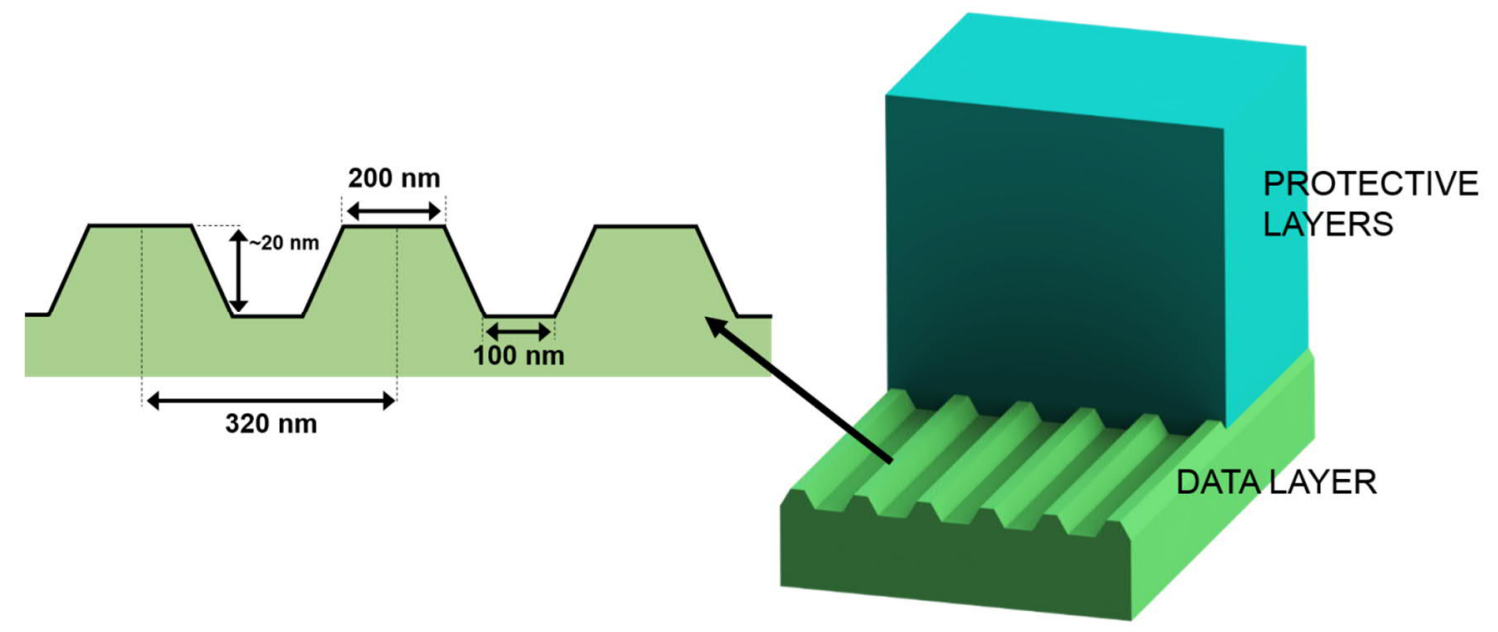

Figure 3. Dimensions of grooved topology of BD-R disc data layer and simplified illustration of removed top layers.

The BD-R disc type used was single time writable $25 \mathrm{~Gb}$ single layer disc (BD-R Datalife 25gb 6x, BD-R-SL:CMCMAGBA5-000, Verbatim). The data was written on disc using standard optical drive (BRXL-PC6U2, Buffalo).

\section{RESULTS}

We define the focus as the intensity maximum along the central axis of the simulation. The focus was determined to be at $1.68 \mu \mathrm{m}$ from the surface of the cylinder, with a width of $0.44 \mu \mathrm{m}$ (FWHM), figure 4 . This result was used to create further simulations, with an aluminum sample placed in the focus of the PNJ. The aluminum sample has a groove, $100 \mathrm{~nm}$ wide and $100 \mathrm{~nm}$ deep. Two simulations were made, with the groove running parallel to (figure 5), and across the axis of the cylinder (figure 6), respectively. 

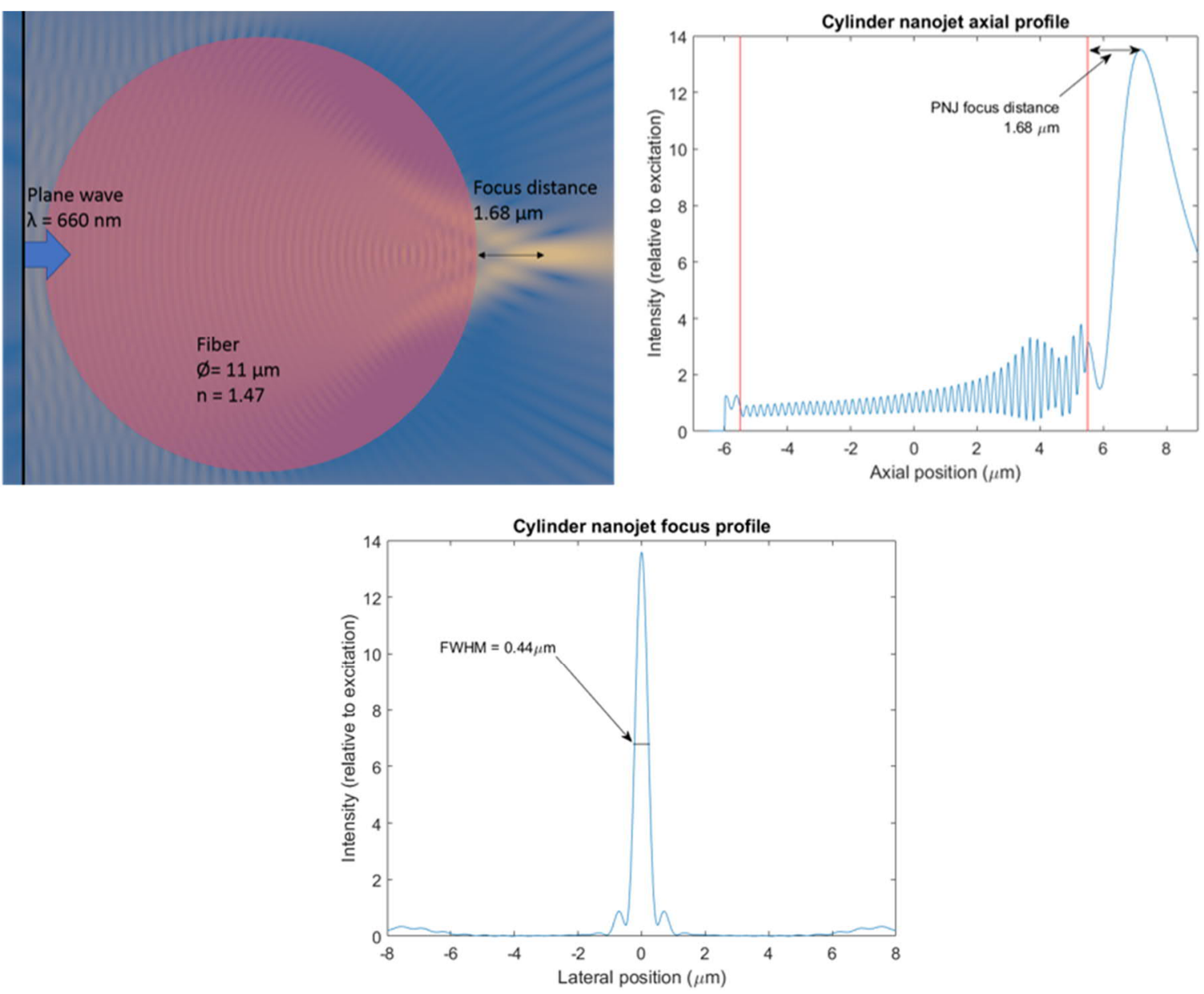

Figure 4. Simulation results of determination of the cylinder focus.
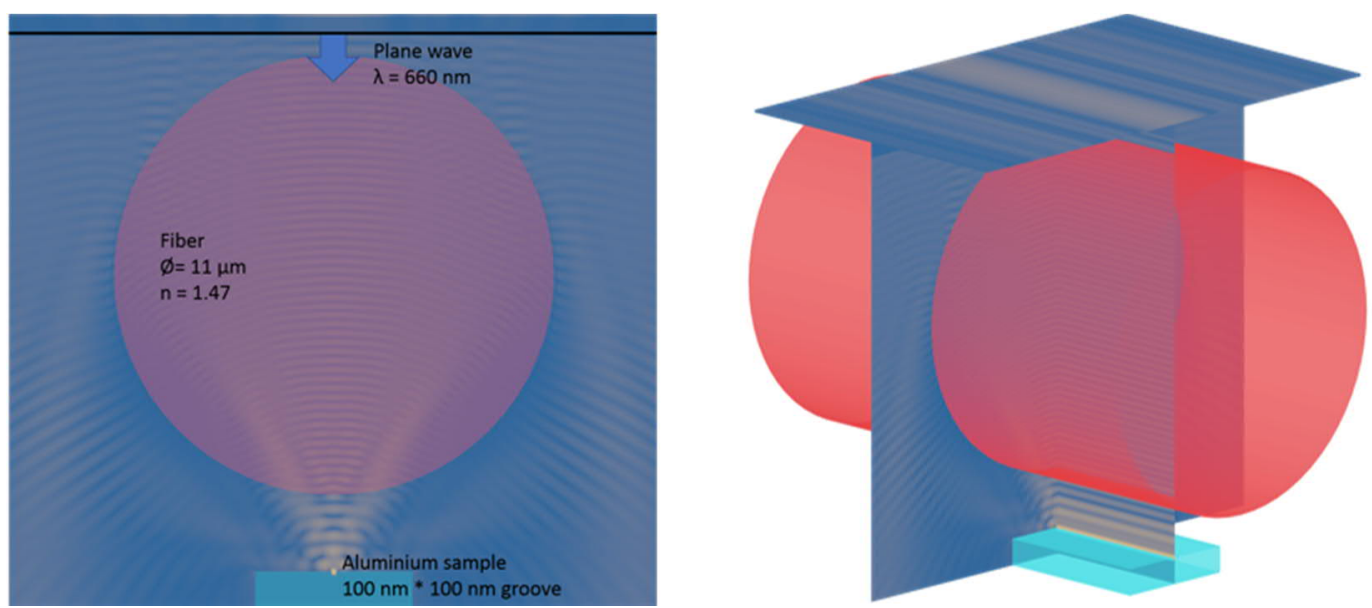

Figure 5. Simulation results when the cylinder is parallel to the groove pattern. 

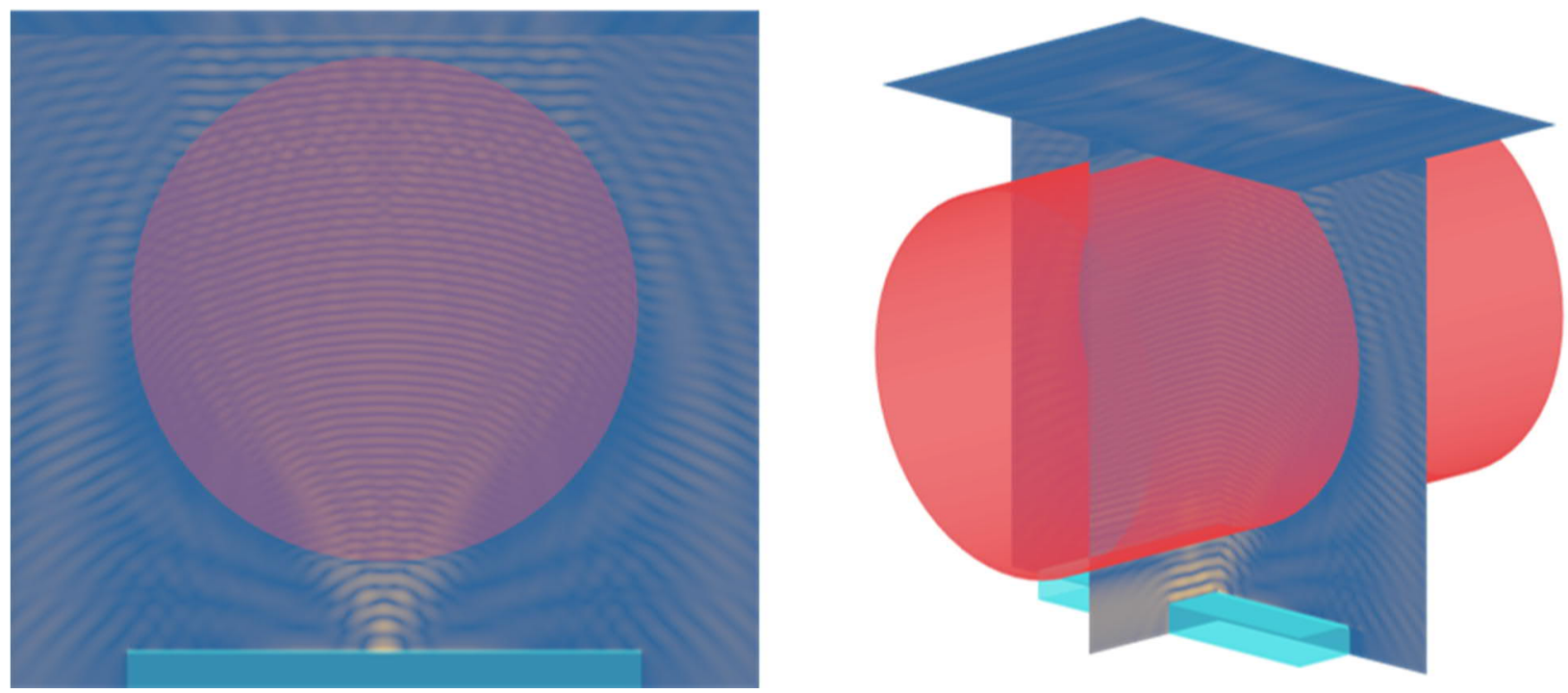

Figure 6. Simulation results when the cylinder is perpendicular to the groove pattern.

The measurement setup using fiber as PNJ creating object imaged the nanometer range topology of BD-R disc data layer. The distinguished grooved structure, following the orientation of data border seen also without PNJ enhancement, could be clearly measured and their 3D topology analyzed. The 2 nd order form removal algorithm was used to remove the aberrations caused by the fiber shape.

The mean pitch of the grooves was measured to be $850 \pm 70 \mathrm{~nm}$ without taking account the extra magnification created by the PNJ object. When compared with the nominal BD-R pitch dimension of $320 \mathrm{~nm}$, the fiber was calculated to create an additional magnification of 2.6 \pm 0.2 , resulting in projected pixel size of below $30 \mathrm{~nm}$. The PNJ magnification corrected effective FOV through the fiber was approximately $28 \times 2 \mu \mathrm{m}$. Figure 7 shows the measured 3D image and profile line through the groove pattern.

The valley-peak-valley heights of the profile illustrated in figure 6 were A: $15.5 \pm 0.8 \mathrm{~nm}, \mathrm{~B}: 9.7 \pm 0.4 \mathrm{~nm}, \mathrm{C}: 10.5 \pm 0.1 \mathrm{~nm}$, and D: $7.2 \pm 0.2 \mathrm{~nm}$. The heights were measured from the leveled lowest position of the valleys (groove bottom) surrounding the measured peak.

\section{DISCUSSION}

Simulations supported the experimentally observed results of using fiber as PNJ creating object. The fiber can be used to focus white light for imaging sample features spatially beyond diffraction limit. The simulations showed that the orientation of the fiber in relation to the imaged feature pattern is not important, and confirmed that imaging features having dimension of $100 \mathrm{~nm}$ is possible using white light. The dimensions obtained through simulations of the optical system of fiber to sample interaction supported the experimental observations.

The AFM measurements obtained during our previous publication about using microsphere as PNJ creating object, showed that exposing the BD-R data layer by peeling the top protective layers away is not perfect. ${ }^{8}$ The top layer is not always peeled entirely leaving regions that might not be topologically consistent. The uneven thickness of layer residues can alter the actual height of the groove topology depending on what region the measurement is taken. To get more constant topology height wise, different sample or more controlled removal method to expose the bare data layer topology should be developed and applied.

The use of fiber as PNJ creating object showed that the phenomenal is not restricted to spherical objects, such as microspheres. We were able to increase the area of FOV by factor of 10x compared to the use of microsphere. 


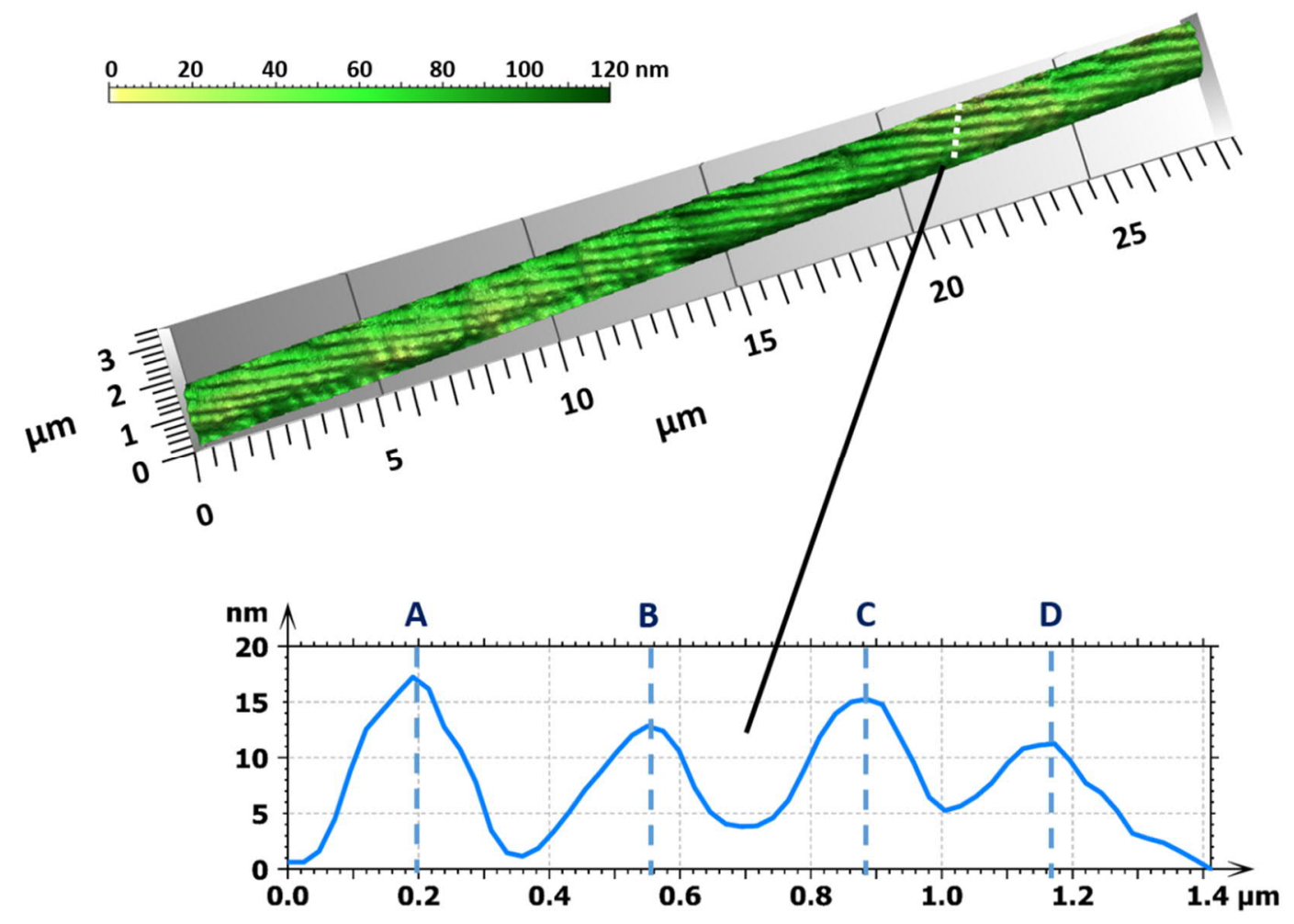

Figure 7. PNJ enhanced large field of view SWLI measurement of BD-R disc data layer topology. The lateral dimensions are shown with PNJ induced magnification correction.

Our measurements show, that the experiments conducted by other groups with obtaining 2D images, can be harnessed with interferometry into 3D super-resolution measurement while still maintaining the large field of view imaging. The optimization of the PNJ object can provide wide development possibilities from the imaging point of view.

\section{CONCLUSIONS}

The PNJ enhanced SWLI was able to resolve super-resolution features of the grooved pattern on a BD-R data layer label free. Imaged features showed that lateral resolution of below $100 \mathrm{~nm}$ was achieved with vertical resolving power of nanometers. Use of fiber as PNJ creating object increased the single image field of view significantly compared to using microsphere as PNJ object. This gives PNJ enhanced SWLI measurement huge advantage compared to currently existing optical super-resolution measurement methods. It can provide label free super-resolution imaging with large field of view.

\section{REFERENCES}

[1] Neice, A., "Methods and Limitations of Subwavelength Imaging," Adv. in Imag. and El. Phys. 163, 117-140 (2010).

[2] Born, M. and Wolf, E. [Principles of Optics], Pergamon, Oxford (1980).

[3] Klar, T. A., Jakobs, S., Dyba, M., Egner, A. and Hell, S. W., "Fluorescence microscopy with diffraction resolution barrier broken by stimulated emission," Proc. Natl. Acad. Sci. 97, 8206-8210 (2000).

[4] Jauß, A., Koenen, J., Weishaupt, K. amd Hollricher, O., "Scanning Near-field Optical Microscopy in Life Science," Single Molecules 3, 232-235 (2002).

[5] Wang, Z., Guo, W., Li, L., Luk'yanchuk, B., Khan, A., Liu, Z., Chen, Z. and Hong, M., "Optical virtual imaging at $50 \mathrm{~nm}$ lateral resolution with a white-light nanoscope," Nature Communications 2, 218 (2011). 
[6] Yang, H., Trouillon, R., Huszka, G. and Gijs, M. A. M., "Super-Resolution Imaging of a Dielectric Microsphere Is Governed by the Waist of Its Photonic Nanojet," Nano Lett.16 (8), pp 4862-4870 (2016).

[7] Kassamakov, I., Lecler, S., Nolvi, A., Leong-Hoï, A., Montgomery, P. and Hæggström, E., "3D Super-Resolution Optical Profiling Using Microsphere Enhanced Mirau Interferometry,” Sci. Rep. 7, 3683 (2017).

[8] Nolvi, A., Hæggström, E., Grundström, K. and Kassamakov, I.. "3D label-free super-resolution imaging," Proc. SPIE 10110, Photonic Instrumentation Engineering IV, 101100L (2017).

[9] Darafsheh, A., Li, Y. and Astratov, V. N., "Super-resolution microscopy by dielectric microcylinders," 15th International Conference on Transparent Optical Networks (ICTON), pp. 1-3 (2013).

[10] De Groot, P., "Principles of interference microscopy for the measurement of surface topography," Advances in Optics and Photonics, 7, 1-65 (2015).

[11] Wyant, J. C., "White light interferometry, " Proc. SPIE 4737, 98-107 (2002).

[12] Schwider, J., Falkenstorfer, O., Screiber, H., Zoller, A. and Streibl, N., "New compensating four-phase algorithm for phaseshift interferometry," Opt. Eng. 32, 1883-1885 (1993).

[13] White Paper: Blu-ray Disc ${ }^{\mathrm{TM}}$ Format, 1. B Physical Format Specifications for BD-R, 5th Edition, Blu-ray Disc Association (2010).

[14] Wang, F., Lianqing L., Peng Y., Zhu L., Haibo Y., Yuechao W. and Wen J. L. "Three-Dimensional Super-Resolution Morphology by Near-Field Assisted White-Light Interferometry,” Sci. Rep. 6, 24703 (2016). 